\title{
Performance verification of Roche Cobas s201 nucleic acid test system in Dongguan Blood Center
}

\author{
Shaobin Chen, Ziyi He", Qingkai Chen, Lin Yu, Qing Wang \\ Dongguan Blood Center, Dongguan, Guangdong, 523930, China
}

\begin{abstract}
In order to verify the performance of the nucleic acid test (NAT) system, the precision and accuracy of the Hamilton STAR pipetting instrument in both single and pool modes were evaluated using pure water simulated for pipetting. The sensitivity, repeatability, anti-interference and anti-contamination ability of the NAT system (Roche Cobas s201) were evaluated using standard serum diluted to different concentrations. The results showed the precisions of the STAR instrument in single and pool modes were $0.07 \%$ and $0.09 \%$, respectively, at the accuracies of $3.33 \%$ and $5.66 \%$. The sensitivity coincidence rate of Roche Cobas s201 was $100 \%$ at the concentration of $3 \times \operatorname{LoD}$ (Limit of Detection). The amplification was inhibited at the concentration of $2 \times$ LoD for the HBV DNA and HCV RNA when the concentration of hemolysis was greater than 7.5\%. Lipemia had no effect, and the anticontamination ability was good. The detection rate in the single mode was higher than the pool mode ( $t=214.3156$, $P<0.01)$. This report suggests that the performance of the NAT system is adequate for laboratory purposes.
\end{abstract}

Keywords: nucleic acid test, performance verification, LoD, Cobas s201

\section{INTRODUCTION}

With the development of nucleic acid amplification technology, increasingly automated and intelligent nucleic acid test (NAT) systems have become widely used for blood screening. For the different principle, reagents and detection modes, a new system is needed to be confirmed before using in laboratory. A previous report by Ohhashi Y et al. examined the Cobas TaqScreen MPX test for use on the Cobas s201 system, showing how important confirmation is for quality control ${ }^{[1]}$. In this report we confirmed the main performance characteristics of the Hamilton STAR and the Roche Cobas s201 system prior to its widespread use.

*Correspondence to: Ziyi He, MD, Department of Clinical Laboratory, Dongguan Blood Center, 19\# Ningjing Road, Humen Town, Dongguan, Guangdong, 523930, China. TEL: 0086-769-85152673, E-mail: zyhe_8@163.com.

Conflict of interests: The authors declared no conflict of interests.

\section{MATERIALS AND METHODS}

\section{Specimen source}

The samples from blood donors with HBsAg, anti HCV, HIV Ab/Ag, anti Treponema pallidum(TP) negative and $\mathrm{ALT} \leqslant 50 \mathrm{U} / \mathrm{mL}$ were tested by the pool mode (pools of 6) using the Roche Cobas s201, from which the results were "no-reaction". The plasma from negative samples without hemolysis, severe lipemia and contamination were selected as negative plasma for dilution. The red bloods cells (RBCs) from negative samples were prepared as RBCs concentrate, and were placed in $-35^{\circ} \mathrm{C}$ to freeze and thawed for 5 minutes for hemolysis prior to use. The lipemia samples (which were chylous lipid blood visible to the naked eye) were placed in $4{ }^{\circ} \mathrm{C}$ for 8 hours, then the upper lipemia plasma was drawn for dilution.

Positive samples were HBV DNA standard serum (Lot NO. 201509001, concentration: $50 \mathrm{U} / \mathrm{mL}$ ), HCV RNA standard serum (Lot NO. 201509001, concentration: 
200 U/mL), HIV-1 RNA (Lot NO. 201509001, concentration: 1,000 U/mL) for blood viruses by NAT, which were provided by Beijing Control \& Standards Biotechnology Co., Ltd (China).

This study was approved by the Ethics Committee of the Dongguan Blood Center and all aspects of the study complied with the Declaration of Helsinki.

\section{Reagents and instruments}

Roche Cobas TaqScreen MPX (v2.0), Roche Cobas s201 (Roche Diagnostics, Switzerland), Microlab STAR IVD (Hamilton, Switzerland), Sorvall ST 40R Centrifuge (Thermo Fisher Scientific, USA), BSA2245S-CW electronic analytical balance (Sartorius Mechatronics T\&H GmbH, Germany), digital temperature and humidity meter were used during the experiment. All the reagents were used within the validity period. All the instruments had been verified or calibrated and were used in normal states. Operation procedures of the sample detection were performed in accordance with the standard operating procedures of the quality management system in our blood center.

\section{Precision and accuracy verification of STAR}

Each sample pool tube (S-tube) was weighed by electronic analytical balance (recorded as $\mathrm{G}_{\text {empty }}$ ), then pure water was used to replace normal samples to pipet for the single test mode (pools of 1) and the pool test mode (pools of 6 ). The temperature and humidity in the lab were recorded when pipetting. Each S-tube was weighed by electronic analytical balance (recorded as $\mathrm{G}_{\text {water }}$ ). The volume of the pure water was calculated by a calculation factor ( $\rho$ water $)$ from density table for different temperature water, which was the volume pipetted from STAR. Finally, the precision and accuracy were calculated and compared with the pipetting parameters. The calculation formula was: the volume pipetted from $\operatorname{STAR}=\left(\mathrm{G}_{\text {water }}-\mathrm{G}_{\text {empty }}\right) / \rho_{\text {water }}$.

\section{Analytical sensitivity verification of Cobas s201}

The analytical sensitivity was denoted by a $95 \%$ limit of detection (LoD), where the mean LoD of HBV DNA, HCV RNA and HIV-1 RNA provided by MPX v2.0 was $2.3 \mathrm{U} / \mathrm{mL}, 6.8 \mathrm{U} / \mathrm{mL}$ and 50.3 $\mathrm{U} / \mathrm{mL}$, respectively. The standard serums of HBV DNA, HCV RNA and HIV-1 RNA were diluted with negative plasma into three concentration $(0.5 \times \mathrm{LoD}$, $1 \times$ LoD and $3 \times$ LoD) groups. The MPX $v 2.0$ was used to perform single tests three times, and two to four pools were tested in each concentration of each batch to calculate the detection rate in each concentration.

\section{Anti-interference verification of Cobas s201}

\section{Hemolytic interference test}

The standard serums of HBV DNA, HCV RNA and HIV-1 RNA were diluted with negative plasma into two concentration $(2 \times$ LoD and $5 \times$ LoD) groups. Each group was mixed with negative RBC samples of 0 (control group), $2.5 \%, 5.0 \%, 7.5 \%$ and $10.0 \%$ volume ratios to simulate hemolysis. MPX v2.0 was used to perform single tests three times, and the CT values in each pool was recorded and compared with the control group.

\section{Lipemia interference test}

The standard serums of HBV DNA, HCV RNA and HIV-l RNA were diluted with lipemia plasma into two concentration $(2 \times \mathrm{LoD}$ and $5 \times \mathrm{LoD})$ groups. MPX v2.0 was used to perform single tests, and each concentration sample was tested twice, from which the $\mathrm{C}_{\mathrm{T}}$ values were compared with the control group.

\section{Anti-contamination verification of Cobas s201}

For single tests, each three copies of standard HBV DNA, HCV RNA, and HIV-1 RNA serums, along with ten negative samples were placed in the order of one positive sample with one negative sample. This was repeated twice and the results of each sample were recorded.

\section{Positive samples pool tests and single tests reproducibility verification of Cobas $\mathbf{2} 201$}

The standard serums of HBV DNA, HCV RNA, and HIV-1 RNA were diluted with negative plasma into five concentration $(0.5 \times \mathrm{LoD}, 1 \times \mathrm{LoD}, 2 \times \mathrm{LoD}, 3 \times \mathrm{LoD}$ and $5 \times \mathrm{LoD}$ ) groups. Both single test mode and pool test mode (each positive sample was mixed with five negative samples) were performed, respectively, and repeated three times to compare the reproducibility.

\section{Statistical analysis}

The SPSS 13.0 statistical software was used for statistical analysis. The $t$ test was used for the data analysis and $P<0.05$ was considered statistically significant.

\section{RESULTS}

\section{Precision and accuracy verification of STAR}

The calculation factor of pure water density ( $\left.\rho_{\text {water }}\right)$ for the STAR was 997.584 (at $22.8^{\circ} \mathrm{C}$ ). The precisions of the single mode and the pool mode were $0.07 \%$ and $0.09 \%$, respectively, and the accuracies were $3.32 \%$ and $5.66 \%$, respectively. The detection rate in the single mode was higher than that in the pool mode $(t=214.3156, P<0.01)$. The results are shown in Table 1. 
Table 1 The pipetting results of each channel for single mode and pool mode in the STAR

\begin{tabular}{lccc}
\hline \multicolumn{1}{c}{ Results } & $\begin{array}{c}\text { Single mode }(\mathrm{mL}) \\
(n=24)\end{array}$ & $\begin{array}{c}\text { Pool mode }(\mathrm{mL}) \\
(n=24)\end{array}$ & $\begin{array}{c}\text { Acceptable } \\
\text { standard }\end{array}$ \\
\hline Measured values & $1.0332 \pm 0.0007$ & $1.0566 \pm 0.0010^{*}$ & \\
$\mathrm{CV}(\%)$ & 0.07 & 0.09 & 2.5 \\
Accuracy $(\%)$ & 3.32 & $5.66^{* *}$ & 5.0 \\
\hline
\end{tabular}

" $t=214.3156, P<0.01$ vs single mode; ${ }^{* *}$ the cumulative departure calculated by the sum of six samples volume in the pool test mode. The accuracy standard of the instrument refers to the accuracy standard in the "instrument calibration report" for instrument calibration given by Roche engineers.

\section{The analytical sensitivity of Cobas s201}

The detection rates of $1 \times$ LoD for HBV DNA and HCV RNA were $80 \%$ and $90 \%$, respectively, which did not meet the experimental requirements. The results were shown in Table 2 .

Table 2 The verification results of the analytical sensitivity for Cobas s201

\begin{tabular}{lccc}
\hline \multirow{2}{*}{ Nucleic acids } & \multicolumn{3}{c}{ Detection rate[Positive No./Total No.(\%)] } \\
\cline { 2 - 4 } & $0.5 \times$ LoD & $1 \times$ LoD & $3 \times$ LoD \\
\hline HIV-1 RNA & $11 / 11(100.00)$ & $10 / 10(100.00)$ & $8 / 8(100.00)$ \\
HBV DNA & $9 / 11(81.82)$ & $8 / 10(80.00)$ & $8 / 8(100.00)$ \\
HCV RNA & $10 / 11(90.91)$ & $9 / 10(90.00)$ & $8 / 8(100.00)$ \\
$\begin{array}{lccc}\text { Acceptable } \\
\text { standard }\end{array}$ & $0 \sim 100 \%$ & $95 \%$ & $100 \%$ \\
\hline
\end{tabular}

\section{Anti-interference verification}

At the concentration of $5 \times \mathrm{LoD}$, with the degree of hemolysis deepened, the $\mathrm{C}_{\mathrm{T}}$ value of detection results gradually increased. At the concentration of $2 \times \mathrm{LoD}$, the amplification of HBV DNA and HCV RNA were suppressed at the concentration of $7.5 \%$ and $10.0 \%$ volume ratio $\mathrm{RBC}$, and the results were non-reactive, as shown in Table 3. Lipemia had no effect for test results, as shown in Table 4.

\section{Anti-contamination verification}

All the results reactive for the HBV DNA, HCV RNA and HIV-1 RNA positive samples were placed according to the order of one positive sample with one negative sample, and the results of the negative samples were non-reactive,. The results are shown in Table 5.

\section{Weakly positive samples pool tests and single tests reproducibility verification}

The pool test results were non-reactive for some HBV DNA, HCV RNA and HIV-1 RNA positive samples at the concentration of $0.5 \times \mathrm{LoD}, 1 \times \mathrm{LoD}$, $2 \times$ LoD and $3 \times$ LoD, but at the concentration of $5 \times \mathrm{LoD}$, all the pool and single test results were reactive, as shown in Table 6.

\section{DISCUSSION}

The confirmation of NAT system is divided into installation qualification, operation qualification and performance qualification according to the implementation process $^{[2]}$. The performance qualification

Table 3 The $\mathrm{C}_{\mathrm{T}}$ values results of different concentration weakly positive samples in different hemolysis

\begin{tabular}{|c|c|c|c|c|c|c|c|c|c|c|}
\hline \multirow{2}{*}{ Nucleic acids } & \multirow{2}{*}{ Concentration } & \multirow{2}{*}{ Control group } & \multicolumn{2}{|c|}{$2.5 \% \mathrm{RBC}$} & \multicolumn{2}{|c|}{$5.0 \% \mathrm{RBC}$} & \multicolumn{2}{|c|}{$7.5 \% \mathrm{RBC}$} & \multicolumn{2}{|c|}{$10.0 \% \mathrm{RBC}$} \\
\hline & & & first & second & first & second & first & second & first & second \\
\hline HIV-1 RNA & $2 \times \mathrm{LoD}$ & 35.3 & 36.2 & 35.2 & 36.2 & 36.7 & 36.4 & 35.9 & 36.9 & 37.0 \\
\hline HBV DNA & $2 \times \operatorname{LoD}$ & 35.8 & 36.0 & 34.8 & 36.0 & 36.3 & 35.4 & - & 35.3 & 46.0 \\
\hline HCV RNA & $2 \times$ LoD & 37.2 & 37.1 & 41.5 & 40.3 & 39.1 & 39.9 & 39.2 & - & - \\
\hline HIV-1 RNA & $5 \times \operatorname{LoD}$ & 33.9 & 33.9 & 33.9 & 33.9 & 35.7 & 35.3 & 35.5 & 36.6 & 36.2 \\
\hline HBV DNA & $5 \times \operatorname{LoD}$ & 34.6 & 33.2 & 33.9 & 33.2 & 33.6 & 33.9 & 33.8 & 34.4 & 36.2 \\
\hline HCV RNA & $5 \times$ LoD & 36.5 & 37.1 & 35.7 & 38.3 & 38.3 & 36.7 & 38.4 & 42.4 & 39.0 \\
\hline
\end{tabular}

Table 4 The $\mathrm{C}_{\mathrm{T}}$ values results of different concentration weakly positive samples for lipemia interference tests

\begin{tabular}{lccccccc}
\hline & \multicolumn{2}{c}{$2 \times$ LOD and lipemia } & & \multicolumn{2}{c}{$5 \times$ LOD and lipemia } \\
\cline { 2 - 4 } Nucleic acids & $\begin{array}{c}\text { Control } \\
\text { group }\end{array}$ & first & second & & $\begin{array}{c}\text { Control } \\
\text { group }\end{array}$ & first & second \\
\hline HIV-1 RNA & 35.3 & 36.0 & 34.8 & & 33.9 & 33.5 & 34.3 \\
HBV DNA & 35.8 & 34.4 & 36.5 & & 34.6 & 33.5 & 33.5 \\
HCV RNA & 37.2 & 37.0 & 37.1 & & 36.5 & 35.5 & 36.2 \\
\hline
\end{tabular}

is based on the expected requirements of technical parameters designed to prove that the new process, equipment or facility can be operated under operating conditions and to provide substantive documentation ${ }^{[3,4]}$. The Roche nucleic acid screening system consists of the Hamilton STAR pipetting instrument and the Cobas s201 nucleic acid test system with the corresponding Cobas TaqScreen MPX v2.0 reagent. The validity and accuracy of the test results are related to the safety of

Table 5 The $\mathrm{C}_{\mathrm{T}}$ values results of single test mode for the negative and positive samples placed according to the order of one positive sample with one negative sample

\begin{tabular}{ccccccccc}
\hline Test No. & $\begin{array}{c}\text { Negative } \\
\text { sample }\end{array}$ & $\begin{array}{c}\text { HIV-1 } \\
\text { RNA+ }\end{array}$ & $\begin{array}{c}\text { Negative } \\
\text { sample }\end{array}$ & HBV DNA+ & $\begin{array}{c}\text { Negative } \\
\text { sample }\end{array}$ & HCV RNA+ & $\begin{array}{c}\text { Negative } \\
\text { sample }\end{array}$ & $\begin{array}{c}\text { HBV DNA+ } \\
\text { sample }\end{array}$ \\
\hline 1 & - & 34.8 & - & 30.4 & - & 33.1 & - & - \\
2 & - & 34.2 & - & 30.6 & - & 32.9 & - \\
\hline
\end{tabular}


Blood Center, 2018, 2(3)

Table 6 The $\mathrm{C}_{\mathrm{T}}$ values results comparison of weakly positive samples pool tests and single tests at different concentration

\begin{tabular}{|c|c|c|c|c|c|c|c|c|c|c|}
\hline \multirow{2}{*}{ Nucleic acids } & \multicolumn{2}{|c|}{$0.5 \times \mathrm{LoD}$} & \multicolumn{2}{|c|}{$1 \times$ LoD } & \multicolumn{2}{|c|}{$2 \times \operatorname{LoD}$} & \multicolumn{2}{|c|}{$3 \times \mathrm{LoD}$} & \multicolumn{2}{|c|}{$5 \times \mathrm{LoD}$} \\
\hline & Single test & $\overline{\text { Pool test }}$ & Single test & Pool test & Single test & Pool test & Single test & Pool test & Single test & Pool test \\
\hline \multirow[t]{3}{*}{ HIV-1 RNA } & 37.4 & - & 36.9 & 37.2 & 35.3 & 38.0 & 34.4 & 35.9 & 33.9 & 35.9 \\
\hline & 37.7 & 40.5 & 38.7 & - & 36.2 & 40.1 & 34.6 & 38.0 & 33.9 & 38.7 \\
\hline & 37.6 & - & 36.9 & - & 35.2 & 37.7 & 35.2 & 38.3 & 33.9 & 37.1 \\
\hline \multirow[t]{2}{*}{ HBV DNA } & 36.8 & 37.2 & 35.0 & - & 35.8 & 36.9 & 34.1 & 37.2 & 34.6 & 36.8 \\
\hline & 36.5 & 38.2 & 34.4 & 43.9 & 36.0 & 38.6 & 33.6 & 36.9 & 33.2 & 36.6 \\
\hline \multirow[t]{3}{*}{ HCV RNA } & 39.0 & 39.4 & 38.4 & - & 37.2 & 39.8 & 37.0 & 37.4 & 36.5 & 38.0 \\
\hline & 38.4 & - & 36.9 & 39.7 & 37.1 & 39.0 & 36.7 & 41.6 & 36.2 & 39.5 \\
\hline & 38.3 & - & 37.7 & - & 37.7 & 39.7 & 36.6 & - & 35.7 & 39.2 \\
\hline
\end{tabular}

clinical transfusion. Therefore, a strict and dependable performance validation is essential. STAR is a main pipetting processing platform, with the main performance parameters being the precision and accuracy in each pipetting channel. We analyzed the pipetting results using pure water to simulate pipetting by electronic analytical balance. The test results showed that all the parameters in STAR single test pipetting mode met the requirements, but the cumulative accuracy of the pool mode was $5.66 \%$, higher than the acceptable standard $\pm 5 \%$. The reason was that a single pipetting volume cannot be individually calculated in the pool test pipetting program. The electronic balance measured the cumulative weight of the pure water after mixing samples from six samples, and the accuracy was the accumulation of six pipetting times, in this way exceeding the acceptable standard for a single sample. This also indicated that the acceptable standard of pipetting volume accuracy for the pool mode should be different from the single pipetting volume. It is recommended to measure each pipetting volume in the pool mode to calculate the average of six pipetting times, then to calculate the total accuracy.

The Cobas s201 is used for qualitative detection in blood screening. The diluted standard serum with negative plasma was used for sensitivity verification. With reference to the MPX v2.0 reagent instructions, the detection of HBV DNA, HCV RNA and HIV-1 RNA pool at the concentration of $0.5 \times \mathrm{LoD}, 1 \times \mathrm{LoD}$, and $2 \times \mathrm{LoD}$ and $3 \times \mathrm{LoD}$ was required. In this way it is not only possible to verify the detection capabilities for the multi-positive samples, but also save reagents. For the test results in Table 2, the detection rates of HBV DNA and HCV RNA at the concentration of $1 \times \operatorname{LoD}$ were lower than $95 \%$, which may have been due to the small base of detected viruses, random and sampling errors and the reduction of sample volume for the pool test. A lower concentration of $0.5 \times \mathrm{LoD}$ can also be reactive, indicating that the sensitivity of the kit was good. Our study reported the same findings as reported by
Ohhashi et al. ${ }^{[1]}$, in which the LoDs for HIV-1 Group M, $\mathrm{HCV}$, and HBV were 49,11 , and $3.8 \mathrm{U} / \mathrm{mL}$, respectively, and 89 and 59.3 copies/mL for HIV-1 Group O and HIV-2, respectively. This was also consistent with the results of Schmidt et $a l^{[5]}$, which reported analytical sensitivities in the range of $1.6 \sim 3.6 \mathrm{U} / \mathrm{mL}, 4.9 \sim 10.9 \mathrm{U} / \mathrm{mL}$ and 14.7 26.6 U/mL for HBV, HCV, and HIV, respectively. In the latest nucleic acid system performance evaluation by Galel et al., sensitivity verification scored better, and $95 \%$ and $50 \%$ detection limits in plasma (U/ $\mathrm{mL}$ ) were 25.7 and 3.8 for HIV-1 Group M, 7.0 and 1.3 for $\mathrm{HCV}$, and 1.4 and 0.3 for $\mathrm{HBV}^{[6]}$.

The quality of nucleic acid samples has a great effect on the results of NAT. In practice, hemolysis and severe lipemia are most common, and they not only impact on pipetting, but also affect test results. We mixed the positive samples with different hemolytic samples and severe lipemia samples to simulate the whole process of routine detection to verify the anti-interference ability of the NAT system. The results showed that mixed positive samples had inhibitory effects on HBV DNA and HCV RNA detection at $2 \times$ LoD concentrations in $7.5 \%$ and $10.0 \%$ hemolysis conditions, which is in accordance with the reagent requirements. While at $5 \times$ LoD concentration, hemolytic positive samples can be detected, but their $\mathrm{C}_{\mathrm{T}}$ values showed a certain fluctuation, and hidden amplification inhibition may have existed. The results of lipemia at the same concentration were not affected, consistent with the results reported by Zhang et al. ${ }^{[7]}$. In the anticontamination tests, the routine single tests were performed according to the order of one positive sample then one negative sample, repeated twice. The results of four positive samples were reactive, and the results of five negative samples were nonreactive, indicating that anti-contamination performance of the NAT system was good in the pipetting and extraction process.

For weakly positive samples, due to a low virus 
concentration in the plasma, it is easy to be missed. In order to verify the compliance of these samples in the single mode and the pool mode of the NAT system, samples close to the LoD concentration were simulated and a comparison of the results of the two modes was carried out. The results showed that the pool mode had missed detection for weakly positive samples at the concentration of less than $5 \times$ LoD. This may be due to a decline in the virus concentration after being mixed with five negative samples, with the virus extract capacity per unit volume decreased resulting in missed detection. The safety of NAT is relative, and our results found the single mode to be better than the pool mode. However, Jongerius et al. showed routine HIV and HCV NAT minipool screening was still able to meet the sensitivity criteria set by the regulatory bodies and provides sufficient specificity and robustness for the timely release of blood donations ${ }^{[8]}$.

There were some deficiencies in the performance verification of the Cobas Roche s201, such as sample management not being validated. Gessoni et al. $.^{[9]} \mathrm{re}-$ ported that samples for NAT testing can be stored until $72 \mathrm{~h}$ at $+4{ }^{\circ} \mathrm{C}$ without any appreciable lowering of the viral-load, which was not taken into account in study. If the laboratory has two sets of the same or different NAT equipment, the performance should be compared ${ }^{[10,11]}$. However, we did not compare the three sets of NAT devices in our laboratory.

In conclusion, the study confirmed the NAT system's performance in a set of test parameters; such as pipetting accuracy, detection sensitivity, anti-interference ability, anti-contamination ability and different test modes. These should be considered and all of the results should meet the requirements. The precision and the accuracy the STAR in single mode are better than those in the pool mode, and the detection rate in the single mode was higher than that in the pool mode. This gives us confidence in clinical application and ensures its suitability. In addition we also found it is very important to evaluate the performance of nucleic acid. Only by continuous performance verification, continuous improvement of quality management can be ensured and achieved.

\section{References}

[1] Ohhashi Y, Pai A, Halait H, et al. Analytical and clinical performance evaluation of the CobasTaqScreen MPX Test for use on the Cobas s201 system. J Virol Methods, 2010,165(2):246-53.

[2] Agnihotri A, Kaur S, Kumar J, et al. Performance qualification: Critical to validation. Asian J Transfus Sci, 2013, 7(1):4-5.

[3] Chevaliez S, Bouvier-Alias M, Laperche S, et al. Performance of version 2.0 of the Cobas AmpliPrep/Cobas TaqMan real-time PCR assay for hepatitis B virus DNA quantification. J Clin Microbiol, 2010, 48(10):3641-7.

[4] ISBT. Guidelines for validation of automated systems in blood establishments. 2nd ed. Vox Sanguinis, 2010,98:1-19.

[5] Schmidt M, Pichl L, Jork C, et al. Blood donor screening with Cobas s 201/CobasTaqScreen MPX under routine conditions at German Red Cross institutes. Vox Sang, 2010, 98(1):37-46.

[6] Galel SA, Simon TL, Williamson PC, et al. Sensitivity and specificity of a new automated system for the detection of hepatitis $\mathrm{B}$ virus, hepatitis $\mathrm{C}$ virus, and human immunodeficiency virus nucleic acid in blood and plasma donations. Transfusion, 2018, 58(3):649-59.

[7] Zhang LM, Yang ZS, Yuan X, et al. Evaluation of the effect of lipid on nucleic acid test results. Chin J Blood Transfusion(in Chinese), 2016, 29(1):55-7.

[8] Jongerius JM, Sjerps M, Cuijpers HT, et al. Validation of the NucliSens Extractor combined with the AmpliScreen HIV version 1.5 and HCV version 2.0 tests for application in NAT minipool screening. Transfusion, 2002, 42(6):792-7.

[9] Gessoni G, Barin P, Valverde S, et al. Biological qualification of blood units: considerations about the effects of sample's handling and storage on stability of nucleic acids. Transfus Apher Sci, 2004, 30(3):197-203.

[10] Stramer SL, Krysztof DE, Brodsky JP, et al. Sensitivity comparison of two Food and Drug Administrationlicensed, triplex nucleic acid test automated assays for hepatitis B virus DNA detection and associated projections of United States yield. Transfusion, 2011, 51(9):2012-22.

[11] Stramer SL, Krysztof DE, Brodsky JP, et al. Comparative analysis of triplex nucleic acid test assays in United States blood donors. Transfusion, 2013, 53(10 Pt 2):2525-37.

(Received 11 June 2018, Revised 19 July 2018, Accepted 23 July 2018) 


\section{Antibodly}

安迪浭㨋

\section{JiangSu LIBO Medicine Biotechnology Co.,Ltd.}

\section{Diagnosis of Hemolytic Disease of the Newborn}

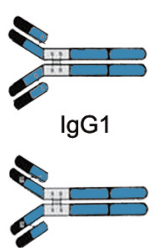

$\lg \mathrm{Ig}$

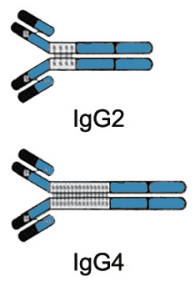

\section{IgG Subclasses Test Card}

The product is used for prediction of Hemolytic Disease of the Newborn, including the detection of Anti-A(B) IgG Subclasses of the Newborn Red Blood Cell and Pregnant Women Serum.

\section{Treatments for Hemolytic Diseases of the Newborn(Fetus) (For Reference Only)}

Pre-pregnancy — Effective Intervention

- Blood typing

- Detection of antibody potency

- Combination of traditional chinese and westem medicine treatment

- Plasmapheresis (Removal)

- High dose of gammaglobulin

\section{Pregnancy — Active Treatment}

- Monitor of antibody potency

- Combination of traditional chinese and westem medicine treatment

- Plasmapheresis (Removal)

- High dose of gammaglobulin

- Intrauterine transfusion

\section{Postpartum — Symptomatic Treatment}

- Hemolysis detection

- Phenobarbital

- Phototherapy

- Gammaglobulin and hormone

- Albumin

- Exchange transfusion in neonates
Early Treatment for Pregnant Women with High Potency

Traditional Chinese Medicine

- Herba Artemisiae Scopariae, Radix Astragali, Fructus Gardeniae

- Herba Taxilli, Semen Cuscutae, Radix Dipsaci Asperoidis

- Radix Angelicae Sinensis, Radix Paeoniae ALBA

- Rhizoma Atractylodis Macrocephalae

\section{Western Medicine}

- Vc: 100mg,1/d;

- VE: $100 \mathrm{mg}, 1 / \mathrm{d}$;

- Oxygen inhalation: 20min per time, 2/d; Plasma(Lymph) Exchange, Immunosorbent Intrauterine Transfusion

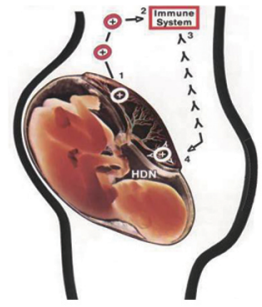

\section{Antibod 进迪溥据 \\ JiangSu LIBO Medicine Biotechnology Co.,Ltd.}

Address: No 78 West Dongsheng Road, Jiangyin, Jiangsu214400, P.R. China

Web Site: www.libiotech.com

Sales Tel: +86-510-86990618、+86-510-86990633

Tech Tel: +86-510-86990608、+86-510-86990655

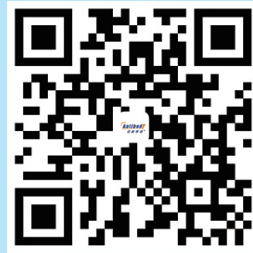

